\title{
HIGHLIGHTS
}

\section{Insights into the kinetics of microRNA biogenesis and turnover}

miRISC
formation
is a kinetic
bottleneck
in miRNA
biogenesis

MicroRNAs (miRNAs) are short non-coding RNAs (ncRNAs) that are essential for animal and plant development and homeostasis; they inhibit gene expression by guiding Argonaute proteins (Ago1 in Drosophila melanogaster) to target mRNAs. Accordingly, miRNA transcription, processing by endoribonucleases and binding by Ago to form the miRNA-induced silencing complex (miRISC) are tightly controlled processes. Reichholf, Herzog et al. now resolve the kinetics of miRNA biogenesis and turnover, and provide valuable new information on miRNA regulation and function.

Individual miRNAs can greatly differ in their stability, from minutes to weeks. To study the kinetics of miRNA expression, the authors employed metabolic labelling by 4-thiouridine ( $4 \mathrm{~s} \mathrm{U})$ coupled with thiol-linked alkylation for the metabolic sequencing of small RNAs (SLAMseq) in fruit fly S2 cells. The fraction of labelled miRNAs significantly increased within minutes of labelling, and production of the 42 most abundant miRNAs was estimated to be $\sim 700$ molecules per minute per cell - a rate considerably higher than previously reported. The steady-state abundance of most, but not all, miRNAs correlated well with their production rate, indicating that production rate is a major but not

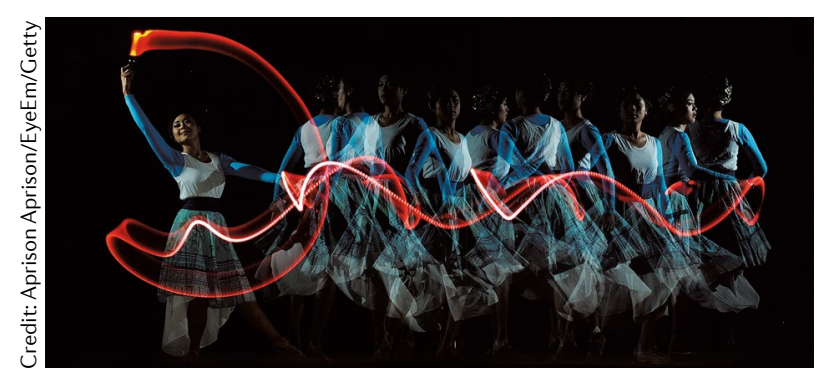

the sole determinant of intracellular miRNA abundance.

Most miRNAs are transcribed as primary miRNAs (pri-miRNAs), which are cleaved to single hairpins termed precursor miRNAs (premiRNAs) and subsequently to shorter, mature miRNA duplexes. Overall, a strong correlation was found between the rates of pri-miRNA transcription and the rates of biogenesis of mature miRNAs.

Of the mature miRNA duplex, one strand ( $\mathrm{miR}$ ) is loaded onto (bound by) Ago and stabilized, whereas the other strand $\left(\mathrm{miR}^{\star}\right)$ is expelled and degraded. The median half-life of the 42 most abundant miR strands was $11.4 \mathrm{~h}$ (although the range was wide), which is almost four times that of mRNAs; by contrast, the median half-life of $\mathrm{miR}^{\star}$ strands was only $\sim 40 \mathrm{~min}$. For some miRNA duplexes, the half-lives of $\mathrm{miR}$ and $\mathrm{miR}^{\star}$ strands were similar, which is indicative of similar ('symmetrical') loading onto miRISC and function in gene silencing.

Kinetic modelling revealed that newly synthesized $\mathrm{miR}$ and $\mathrm{miR}^{\star}$ strands accumulated at very similar rates, reflecting the rapid biogenesis of miRNA duplexes. At later time points, when most $\mathrm{miR}^{\star}$ strands have been degraded, miR strand accumulation also slowed down owing to an estimated decay of $40 \%$ of miRNA duplexes taking place even before Ago loading.

Importantly, moderate increase in Ago1 levels correlated with increase in miRNA abundance, indicating that miRISC formation is a kinetic bottleneck in miRNA biogenesis and function. Furthermore, overexpression of an Agol transgene was accompanied by a decrease in the levels of endogenous Agol proteins, suggesting that limiting the abundance of Agol helps to restrict the loading of miRISC to miRNAs, which are the functionally suitable partners of Ago1, at the expense of the abundant decay intermediates of other ncRNAs. Indeed, sequencing of Ago-bound small RNAs revealed an increase in ribosomal RNA, tRNA, small nuclear RNA and small nucleolar RNA relative to miRNAs upon Ago1 overexpression.

The addition or trimming of nucleotides (mostly uridines) to the 3 '-end of pre-miRNAs and mature miRNAs produces isomiRs and can affect pre-miRNA processing and miRNA function, respectively. For example, in flies, mature miR-34-5p has several isomiRs owing to the activity of the 3'-to-5' exoribonuclease Nibbler. Trimming of 4sU-labelled miR-34-5p co-occurred with the selective stabilization of miR-34-5p relative to $\mathrm{miR}-34-3 p$ (the $\mathrm{miR}^{\star}$ strand), indicating that trimming of $\mathrm{miR}$ strands follows $\mathrm{miR}^{\star}$ removal and Ago1 loading. Furthermore, 25 miRNAs were identified as Nibbler substrates and were considerably less stable compared with total miRNAs, suggesting that $3^{\prime}$-end processing promotes miRNA turnover.

In summary, miRNAs are overall highly produced and have long halflives, which explains their high cellular abundance and effectiveness in mRNA targeting. Finely tuned Ago expression supports the selectivity of loading miRNAs over other small ncRNAs, and miRISC assembly is important for the stability of mature miRNAs.

Eytan Zlotorynski

ORIGINAL ARTICLE Reichholf, B. et al.

Time-resolved small RNA sequencing unravels the molecular principles of microRNA homeostasis. Mol. Cell https://doi.org/10.1016/ j.molcel.2019.06.018 (2019) FURTHER READING Treiber, T. et al. Regulation of microRNA biogenesis and its crosstalk with other cellular pathways. Nat. Rev. Mol. Cell Biol. 20, 5-20 (2019)|Herzog, V. A. et al. Thiol-linked alkylation of RNA to assess expression dynamics. Nat. Methods 14, 1198-1204 (2017) 